University of Puget Sound

Sound Ideas

All Faculty Scholarship

Faculty Scholarship

4-1-1987

\title{
The Operation of Maxwell's Demon in a Low Entropy System
}

Andrew Rex

University of Puget Sound, rex@pugetsound.edu

Follow this and additional works at: http://soundideas.pugetsound.edu/faculty_pubs

\section{Citation}

Rex, A F. "The Operation of Maxwell's Demon in a Low Entropy System." American Journal of Physics. 55.4 (1987). Print.

This Article is brought to you for free and open access by the Faculty Scholarship at Sound Ideas. It has been accepted for inclusion in All Faculty Scholarship by an authorized administrator of Sound Ideas. For more information, please contact soundideas@pugetsound.edu. 


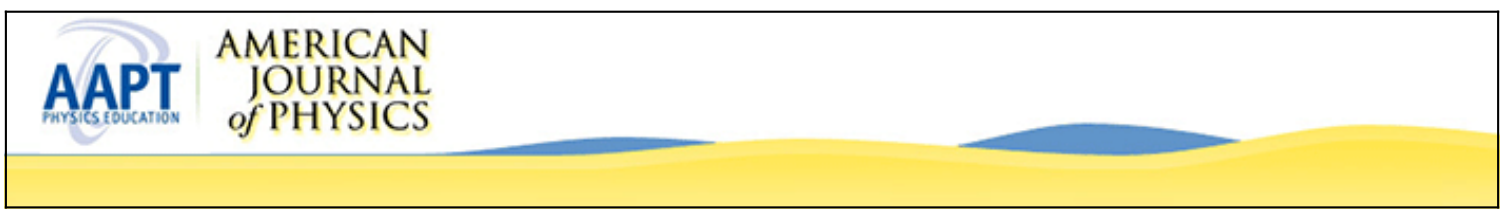

The operation of Maxwell's demon in a low entropy system

A. F. Rex

Citation: American Journal of Physics 55, 359 (1987); doi: 10.1119/1.15171

View online: http://dx.doi.org/10.1119/1.15171

View Table of Contents: http://scitation.aip.org/content/aapt/journal/ajp/55/4?ver=pdfcov

Published by the American Association of Physics Teachers

Articles you may be interested in

Engineering Maxwell's demon

Phys. Today 67,60 (2014); 10.1063/PT.3.2490

Unmasking Maxwell's Demon

AIP Conf. Proc. 643, 436 (2002); 10.1063/1.1523841

Maxwell's Demon: Entropy, Information, Computing

Am. J. Phys. 60, 282 (1992); 10.1119/1.16914

Maxwell's Demon

Phys. Teach. 13, 503 (1975); 10.1119/1.2339244

Maxwell's Demon Cannot Operate: Information and Entropy. I

J. Appl. Phys. 22, 334 (1951); 10.1063/1.1699951

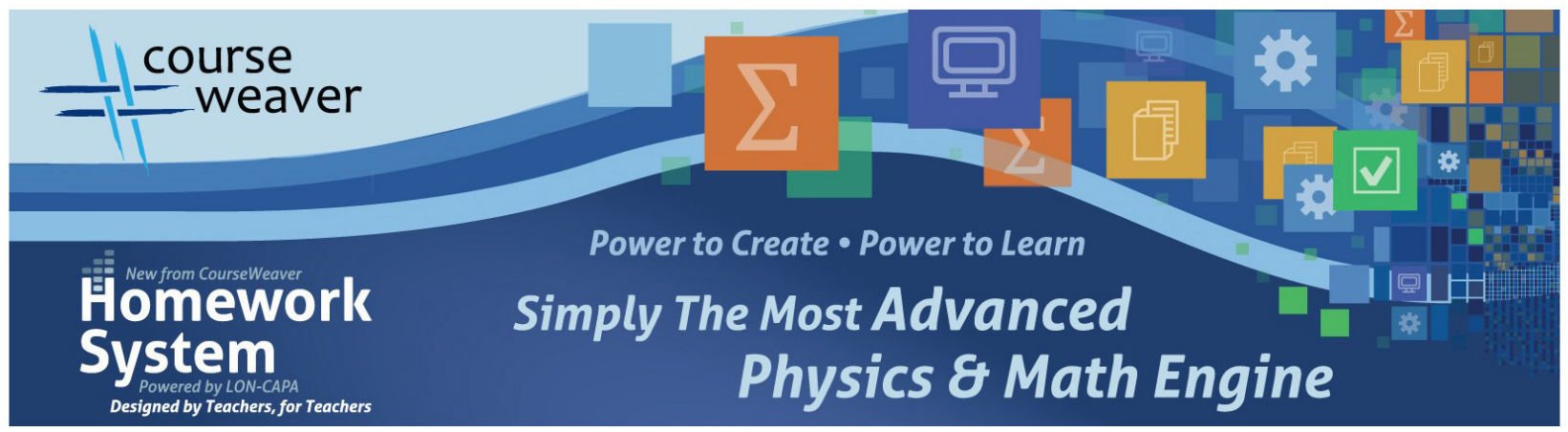


of Illinois Public Act 83-613 (Satterthwaite Bill) Illinois Board of Higher Education.

${ }^{1}$ Canberra Laboratory Manual (Canberra Industries, Inc., 1977). Experiments in Nuclear Science (Ortec, Inc., 1976).

${ }^{2}$ G. I. Gleason, Isotopic Neutron Source Experiments, ORAU-102, Oak Ridge Associated Universities, 1967.

${ }^{3}$ Model TEL NU500 from Tel-Atomic, Inc., Model S441064 from Fisher Scientific, and Model 30191 from Central Scientific Co.

${ }^{4}$ Geiger-Muller tube from the Nucleus, also used in their Model 500.

${ }^{5}$ Ortec, Inc., Model 467.

'Nuclear Data, Model 60.
${ }^{7}$ A. C. Melissinos, Experiments in Modern Physics (Academic, New York, 1966), p. 187.

${ }^{8}$ Through a cylindrical water moderator of $30 \mathrm{~cm}$ diam $\times 50 \mathrm{~cm}$ height with the source in the center the surface strength is $0.5 \mathrm{mr} / \mathrm{h}$ of gamma activity and $0.7 \mathrm{mr} / \mathrm{h}$ of neutron activity as measured with a Victoreen Model 492 Survey Meter and a Victoreen Model 478 Portable Neutron Monitor.

${ }^{9}$ E. Segré, Experimental Nuclear Physics (Wiley, New York, 1953), Vol. II, p. 397.

${ }^{10}$ The fit was done with the program, DESCALC, by J. D. Larson. Although widely circulated the program is not supported by Brookhaven National Laboratory. Most scientific subroutine libraries have a similar program.

"S. F. Mughabghab, M. Divadeenam, and N. E. Holden, Neutron Cross Sections (Academic, New York, 1981), Vol. I, Part A, pp. 47-5 and 477.

\title{
The operation of Maxwell's demon in a low entropy system
}

\author{
A. F. Rex \\ Physics Department, University of Puget Sound, Tacoma, Washington 98416
}

(Received 24 October 1985; accepted for publication 28 March 1986)

\begin{abstract}
The problem of Maxwell's sorting demon traditionally has been studied for the case in which the hot and cold regions differ very little in temperature. In this article a solution is presented for the case in which the temperature difference is great so that the total entropy is lower. Calculations indicate that in this case the demon must use a large number of photons to observe the proper kinds of particles. This causes an increase in entropy which more than offsets the decrease caused by an exchange of particles.
\end{abstract}

\section{INTRODUCTION}

The well-known sorting demon first proposed by Maxwell ${ }^{1}$ remains one of the best examples of the wide range of applicability of the second law of thermodynamics. Maxwell was not able to reconcile the demon completely with the second law because he failed to take into account the energy (and hence the entropy) added to a system of particles by the act of observation. The necessary understanding of light quantization and black body radiation was not available at the time of Maxwell's death in 1879. Some relevant work concerning the relationship between quantum mechanics and the second law was done by Born and Green. ${ }^{2,3}$ However, a more straightforward approach was taken by Brillouin, 4,5 who used only the "old" quantum theory of light quantization and black body radiation, along with classical thermodynamics. It is this approach to the problem of Maxwell's demon which is cited most often (although still infrequently) by thermodynamics texts and monographs. ${ }^{6}$

Brillouin's solution is valid over almost the entire range of classical thermodynamics. However, there are some particularly interesting examples with parameters lying outside of those allowed by Brillouin's simplifying assumptions. This article will consider some of those examples. Whenever necessary the most generous concessions will be allowed the demon. In spite of these concessions, the de- mon (not surprisingly) still will not be able to violate the second law.

\section{THEORY}

Brillouin considered two gas-filled regions $A$ and $B$ separated by a movable partition. The partition is controlled by the demon, who hopes to decrease the total entropy of the system by allowing only relatively fast particles to pass from the cold to the hot region. It is assumed that $T_{B}>T_{A}$, with $T_{B}-T_{A}=\Delta T$. Also, a temperature $T$ is defined so that

$$
T_{A}=T-\frac{1}{2} \Delta T
$$

and

$$
T_{B}=T+\frac{1}{2} \Delta T \text {. }
$$

In each region $j$ the equipartition theorem dictates that the mean energy of a particle is

$$
\bar{E}_{j}=\frac{3}{2} k T_{j} \text {. }
$$

Therefore the demon should select a particle from $A$ with energy $\frac{3}{2} k T\left(1+\epsilon_{1}\right)$ and allow it to pass into $B$. Similarly, a particle with energy $\frac{3}{2} k T\left(1-\epsilon_{2}\right)$ will be allowed to pass from $B$ into $A$.

In order to see those particles, one must distinguish photons scattered from them from the black body radiation. 
This requires that the energy of the photon used to observe the particle is $h v>k T$. The entropy increase due to the act of observation is then

$$
\Delta S_{d}=h v / T=k b \text {, }
$$

where $b=h v / k T>1$. Since (at least) two photons must be used in the process described above, the net entropy increase is

$$
\Delta S_{d}=2 k b .
$$

The entropy decrease effected by the demon is calculated using the energy transfer

$$
Q=\frac{3}{2} k T\left(\epsilon_{1}+\epsilon_{2}\right) \text {. }
$$

The resulting entropy decrease is

$$
\Delta S_{i}=\left(Q / T_{B}\right)-\left(Q / T_{A}\right) \cong-Q(\Delta T) / T^{2}
$$

or

$$
\Delta S_{i} \cong-\frac{3}{2} k\left(\epsilon_{1}+\epsilon_{2}\right)(\Delta T / T) .
$$

Now Brillouin presumes that both $\epsilon_{1}$ and $\epsilon_{2}$ are small and that $\Delta T \ll T$, which means that

$$
\Delta S_{i}=-\frac{3}{2} k \eta,
$$

with $\eta \ll 1$. Therefore the net entropy change is

$$
\Delta S_{d}+S_{i} \cong k\left(2 b-\frac{3}{2} \eta\right)>0
$$

and the second law is validated.

The example just presented is not valid universally, however. Crucial to this argument is the assumption in Eq. (7) that $T_{A} T_{B} \cong T^{2}$. The exact expression is

$$
T_{A} T_{B}=T^{2}-(\Delta T)^{2} / 4
$$

The resulting entropy decrease due to energy exchange is now

$$
\Delta S_{i}=-Q(\Delta T) /\left[T^{2}-(\Delta T)^{2} / 4\right] .
$$

$\Delta S_{i}$ is not necessarily negligible any more. In the limit as
$\Delta T$ approaches $T$ (the most favorable case from the demon's point of view),

$$
\Delta S_{i}=-\frac{3}{4} k\left(\epsilon_{1}+\epsilon_{2}\right)\left[\left(T_{B}^{2}-T_{A}^{2}\right) /\left(T_{A} T_{B}\right)\right],
$$

or using Eq. (6),

$$
\Delta S_{i} \cong-\frac{3}{4} k\left(\epsilon_{1}+\epsilon_{2}\right)\left(T_{B} / T_{A}\right) .
$$

A comparison of Eqs. (5) and (14) reveals where the difficulty lies. By definition $\epsilon_{2}<1$, but in principle $\epsilon_{1}$ may be somewhat greater than unity. At first glance this would seem to be a violation of the second law, for while $b$ must be greater than unity in order to distinguish a particle from the black body radiation, there is no clear justification for it to be greater than $\epsilon_{1}$.

The resolution of this problem lies in the fact that a single photon will not be sufficient to find a particle with the desired velocity. Consider how the values of $\epsilon_{1}$ relate to the physical situation. In order to obtain a relatively high value for $\epsilon_{1}$ (even to obtain a value approaching unity), the demon must select a particle with exceptionally high speed compared with the mean.

$$
\bar{v}_{A}=\sqrt{8 k T_{A} / \pi m},
$$

where $m$ is the mass of a single particle. Particles with this desired characteristic will be found only far out on the

\begin{tabular}{|c|c|c|c|c|c|c|c|c|c|}
\hline$T_{A}$ & $T_{B}$ & $\bar{v}_{A}$ & $\bar{v}_{B}$ & $v_{1}$ & $v_{2}$ & $n_{1}$ & $n_{2}$ & $\epsilon_{1}$ & $\epsilon_{2}$ \\
\hline 300 & 320 & 475 & 490 & 300 & 600 & 3.9 & 5.3 & 0.31 & 0.67 \\
\hline 300 & 320 & 475 & 490 & 100 & 800 & 16 & 113 & 1.3 & 0.96 \\
\hline 300 & 320 & 475 & 490 & 100 & 1200 & 1100 & 113 & 4.3 & 0.96 \\
\hline 200 & 220 & 388 & 407 & 300 & 500 & 4.2 & 3.4 & 0.34 & 0.52 \\
\hline 200 & 220 & 388 & 407 & 200 & 600 & 9.4 & 9.3 & 0.94 & 0.78 \\
\hline 200 & 220 & 388 & 407 & 200 & 1000 & 1500 & 9.3 & 4.4 & 0.78 \\
\hline 100 & 120 & 274 & 300 & 200 & 400 & 7.0 & 4.3 & 0.64 & 0.59 \\
\hline 100 & 120 & 274 & 300 & 100 & 500 & 27 & 27 & 1.6 & 0.90 \\
\hline 100 & 120 & 274 & 300 & 100 & 1000 & $7.2 \times 10^{6}$ & 27 & 9.3 & 0.90 \\
\hline 600 & 620 & 672 & 683 & 600 & 800 & 3.3 & 2.4 & 0.19 & 0.33 \\
\hline 600 & 620 & 672 & 683 & 400 & 1000 & 7.7 & 5.9 & 0.85 & 0.70 \\
\hline 600 & 620 & 672 & 683 & 400 & 2000 & $2.4 \times 10^{4}$ & 5.9 & 6.4 & 0.70 \\
\hline 2000 & 2100 & 1226 & 1256 & 1000 & 2000 & 13 & 2.9 & 1.2 & 0.45 \\
\hline 2000 & 2100 & 1226 & 1256 & 1000 & 3000 & 670 & 2.9 & 4.0 & 0.45 \\
\hline 300 & 500 & 475 & 612 & 300 & 800 & 16 & 9.5 & $0: 81$ & 0.75 \\
\hline 300 & 500 & 475 & 612 & 300 & 1200 & 1100 & 7.5 & 3.1 & 0.75 \\
\hline 300 & 1000 & 475 & 867 & 300 & 1200 & 1100 & 25 & 1.5 & 0.84 \\
\hline 300 & 1000 & 475 & 867 & 200 & 2000 & $2.4 \times 10^{9}$ & 79 & 6.0 & 0.93 \\
\hline 200 & 500 & 388 & 613 & 200 & 800 & 82 & 29 & 1.1 & 0.87 \\
\hline 200 & 500 & 388 & 613 & 200 & 1250 & $6.0 \times 10^{4}$ & 29 & 3.7 & 0.87 \\
\hline 100 & 500 & 274 & 613 & 100 & 800 & $1.6 \times 10^{4}$ & 220 & 1.4 & 0.96 \\
\hline 100 & 500 & 274 & 613 & 100 & 1000 & $7.2 \times 10^{6}$ & 220 & 2.8 & 0.96 \\
\hline
\end{tabular}
"tail" of the Maxwell speed distribution. The fraction $F$ of those particles with a speed greater than a given speed $v_{2}$ for a classical gas at temperature $T_{A}$ is

$$
F=\int_{v_{2}}^{\infty} f(v) d v
$$

where $f(v)$ is the normalized Maxwell function

$$
f(v)=4\left(m / 2 \pi k T_{A}\right)^{3 / 2} v^{2} \exp \left(-m v^{2} / 2 k T_{A}\right) .
$$

This means that the number $n_{1}$ of photons normally re-

Table I. Calculated values of $n_{1}, n_{2}, \epsilon_{1}$, and $\epsilon_{2}$ as a function of temperatures and selected speeds for nitrogen gas. Temperatures are in $\mathrm{K}$ and speeds in $\mathrm{m} / \mathrm{s}$. 
Table II. Calculated values of $n_{1}, n_{2}, \epsilon_{1}$, and $\epsilon_{2}$ as a function of temperatures and selected speeds for hydrogen gas. Temperatures are in $\mathrm{K}$ and speeds in $\mathrm{m} / \mathrm{s}$.

\begin{tabular}{rrrrrrrrrr}
\hline$T_{A}$ & $T_{B}$ & $\bar{v}_{A}$ & $\bar{v}_{B}$ & $v_{1}$ & $v_{2}$ & $n_{1}$ & $n_{2}$ & $\epsilon_{1}$ \\
\hline 300 & 320 & 1777 & 1835 & 1500 & 2100 & 3.2 & 2.8 & 0.15 & 0.41 \\
300 & 320 & 1777 & 1835 & 1000 & 2500 & 6.0 & 7.1 & 0.63 & 0.74 \\
300 & 320 & 1777 & 1835 & 1000 & 3000 & 16 & 7.1 & 1.3 & 0.74 \\
300 & 320 & 1777 & 1835 & 1000 & 3500 & 53 & 7.1 & 2.2 & 0.74 \\
200 & 220 & 1451 & 1521 & 1200 & 2000 & 5.5 & 3.0 & 0.54 & 0.45 \\
200 & 220 & 1451 & 1521 & 1000 & 3000 & 84 & 4.5 & 2.5 & 0.62 \\
30 & 50 & 562 & 725 & 300 & 1000 & 23 & 15 & 1.0 & 0.82 \\
30 & 50 & 562 & 725 & 300 & 1500 & 2800 & 15 & 3.5 & 0.82 \\
600 & 620 & 2513 & 2554 & 2000 & 3000 & 3.3 & 3.0 & 0.19 & 0.47 \\
600 & 620 & 2513 & 2554 & 2000 & 5000 & 58 & 3.0 & 2.3 & 0.47 \\
2000 & 2100 & 4587 & 4701 & 4000 & 6000 & 4.5 & 2.5 & 0.42 & 0.37 \\
2000 & 2100 & 4587 & 4701 & 4000 & 10000 & 150 & 350 & 2.9 & 0.37 \\
300 & 1000 & 1777 & 3244 & 1500 & 3500 & 53 & 11 & 0.52 & 0.72 \\
300 & 1000 & 1777 & 3244 & 1000 & 5000 & 7400 & 34 & 2.1 & 0.88 \\
200 & 1000 & 1451 & 3244 & 1400 & 3500 & 550 & 13 & 0.65 & 0.74 \\
200 & 1000 & 1451 & 3244 & 1400 & 5000 & $1.1 \times 10^{6}$ & 13 & 2.4 & 0.74 \\
100 & 1000 & 1026 & 3244 & 1000 & 3300 & $1.6 \times 10^{5}$ & 34 & 0.60 & 0.85 \\
100 & 1000 & 1026 & 3244 & 1000 & 3500 & $8.4 \times 10^{5}$ & 34 & 0.80 \\
\hline \hline
\end{tabular}

quired to find a particle with speed greater than $v_{2}$ will be

$$
n_{1}=\frac{1}{F}=\left(\int_{v_{2}}^{\infty} f(v) d v\right)^{-1} \text {. }
$$

Similarly the demon must select an appropriately slow particle for transfer from $B$ to $A$. Clearly the number of photons needed to find such a particle (with speed less than $v_{1}$ ) will be

$$
n_{2}=\left(\int_{0}^{v_{1}} f(v) d v\right)^{-1}
$$

Note that the temperature $T_{B}$ must be used in $f(v)$ in Eq. (19). Also, it is clear that $v_{1}$ must not be more than $\bar{v}_{A}$ and $v_{2}$ must not be less than $\bar{v}_{B}$. With these constraints the net entropy change will be

$$
\Delta S_{d}+\Delta S_{i} \geqslant k\left(n_{1} b+n_{2} b-2 \epsilon_{1}-2 \epsilon_{2}\right),
$$

where the inequality is due to the nature of the approximations made to obtain Eqs. (13) and (14). The sum of $n_{1}$ and $n_{2}$ must be sufficiently large in order for (20) to satisfy the second law, i.e., so that the right-hand side of (20) is positive.

\section{RESULTS}

The integrals in Eqs. (18) and (19) were performed on a computer using Newtonian quadrature. The integral in Eq. (18) was calculated using limits $v_{2}$ to $10 v_{2}$. The exponential factor in the Maxwell function ensures that these limits will be sufficient to yield a very accurate result.

Tables I and II show some results for the typical gases nitrogen and hydrogen. Since $\epsilon_{2}$ is restricted to values $0<\epsilon_{2}<1$, it is useful to concentrate on $\epsilon_{1}$. As was noted in Sec. II, higher values of $\epsilon_{1}$ represent a greater entropy de-

\begin{tabular}{|c|c|c|c|c|c|c|c|c|c|}
\hline$T_{A}$ & $T_{B}$ & $\bar{v}_{A}$ & $\bar{v}_{B}$ & $v_{1}$ & $v_{2}$ & $n_{1}$ & $n_{2}$ & $\epsilon_{1}$ & $\epsilon_{2}$ \\
\hline 300 & 320 & $1.08 \times 10^{5}$ & $1.12 \times 10^{5}$ & $1.0 \times 10^{5}$ & $1.2 \times 10^{5}$ & 2.7 & 2.3 & 0.01 & 0.30 \\
\hline 300 & 320 & $1.08 \times 10^{5}$ & $1.12 \times 10^{5}$ & $1.0 \times 10^{5}$ & $2.0 \times 10^{5}$ & 31 & 2.3 & 1.8 & 0.30 \\
\hline 300 & 320 & $1.08 \times 10^{5}$ & $1.12 \times 10^{5}$ & $1.0 \times 10^{5}$ & $3.0 \times 10^{5}$ & 5600 & 2.3 & 5.3 & 0.30 \\
\hline 100 & 120 & $6.2 \times 10^{4}$ & $6.8 \times 10^{4}$ & $5.0 \times 10^{4}$ & $1.0 \times 10^{5}$ & 11 & 3.5 & 0.98 & 0.50 \\
\hline 100 & 120 & $6.2 \times 10^{4}$ & $6.8 \times 10^{4}$ & $5.0 \times 10^{4}$ & $2.0 \times 10^{5}$ & $1.4 \times 10^{5}$ & 3.5 & 6.9 & 0.50 \\
\hline 1 & 2 & 6200 & 8800 & 5000 & $1.5 \times 10^{4}$ & 515 & 6.5 & 2.3 & 0.64 \\
\hline 1 & 2 & 6200 & 8800 & 1900 & $2.0 \times 10^{4}$ & $1.4 \times 10^{5}$ & 6.5 & 4.8 & 0.64 \\
\hline 0.1 & 1 & 1974 & 6242 & 1900 & 6500 & $3.0 \times 10^{5}$ & 35 & 0.67 & 0.86 \\
\hline 0.1 & 1 & 1974 & 6242 & 1900 & $1.0 \times 10^{4}$ & $9.5 \times 10^{13}$ & 35 & 3.0 & 0.86 \\
\hline $1.0 \times 10^{-6}$ & $2.0 \times 10^{-6}$ & 6.2 & 8.8 & 6 & 10 & 12 & 4.1 & 0.45 & 0.48 \\
\hline $1.0 \times 10^{-6}$ & $2.0 \times 10^{-6}$ & 6.2 & 8.8 & 6 & 20 & $1.4 \times 10^{5}$ & 4.1 & 4.8 & 0.48 \\
\hline 1000 & 1500 & $2.0 \times 10^{5}$ & $2.4 \times 10^{5}$ & $1.9 \times 10^{5}$ & $2.5 \times 10^{5}$ & 4.0 & 3.0 & 0.09 & 0.37 \\
\hline 1000 & 1500 & $2.0 \times 10^{5}$ & $2.4 \times 10^{5}$ & $1.9 \times 10^{5}$ & $3.0 \times 10^{5}$ & 8.6 & 3.0 & 0.57 & 0.37 \\
\hline 1000 & 1500 & $2.0 \times 10^{5}$ & $2.4 \times 10^{5}$ & $1.9 \times 10^{5}$ & $4.0 \times 10^{5}$ & 69 & 3.0 & 1.8 & 0.37 \\
\hline 1000 & 2000 & $1.0 \times 10^{5}$ & $2.8 \times 10^{5}$ & $1.9 \times 10^{5}$ & $3.0 \times 10^{5}$ & 8.6 & 4.1 & 0.31 & 0.48 \\
\hline 1000 & 2000 & $2.0 \times 10^{5}$ & $2.8 \times 10^{5}$ & $1.9 \times 10^{5}$ & $4.0 \times 10^{5}$ & 69 & 4.1 & 1.3 & 0.48 \\
\hline
\end{tabular}

Table III. Calculated values of $n_{1}, n_{2}, \epsilon_{1}$, and $\epsilon_{2}$ as a function of temperatures and selected speeds for a dilute gas of electrons. Temperatures are in $\mathrm{K}$ and speeds in $\mathrm{m} / \mathrm{s}$. 
crease associated with the exchange of particles between $A$ and $B$. The calculations presented in Tables I and II suggest strongly that there is no adjustment of parameters which will result in a favorable outcome for the demon, i.e., a net decrease in entropy. The best strategy for increasing $\epsilon_{1}$, namely to increase $v_{2}$, is precisely the thing which causes $n_{1}$ to increase most quickly. In all cases examined it is clear that $n_{1}$ increases much more rapidly than $\epsilon_{1}$. This should not be unexpected considering the nature of the statistical distribution of speeds.

In order to determine whether this result is peculiar to typical gas molecules, calculations were also performed for a gas of electrons. It is assumed that the gas is dilute enough so that Coulomb forces do not adversely affect the work of the demon. This assumption also makes it reasonable to believe that classical statistics will be valid over the wide range of temperatures considered. Again the results (shown in Table III) indicate that any attempt to increase $\epsilon_{1}$ results in a much greater increase in $n_{1}$. This is true for a wide range of temperatures $T_{A}$ and $T_{B}$.

While these results are by no means exhaustive, they provide further insight into the problems faced by Maxwell's entropy reducing demon. Indeed it is clear that these entropy reductions can only be slight in comparison with the entropy added through the required process of observation. It is interesting to note what a crucial role this process of observation (normally important in modern quantum theory) plays in dealing with particles which obey classical statistics. This is perhaps one of the best examples of such an overlap between classical and quantum concepts.

'J. C. Maxwell, Theory of Heat (London, 1871).

${ }^{2}$ M. Born and H. S. Green, Proc. R. Soc. London Ser. A 192, 166 (1948).

${ }^{3}$ Max Born, Ann. Phys. 3, 107 (1948).

${ }^{4}$ L. Brillouin, J. Appl. Phys. 22, 334 (1951).

${ }^{5}$ L. Brillouin, J. Appl. Phys. 22, 338 ( 1951).

'See, for example, A. B. Pippard, The Elements of Classical Thermodynamics (Cambridge U. P., Cambridge, 1981).

\title{
The gravitational Poynting vector and energy transfer
}

\author{
Peter Krumm and Donald Bedford \\ Department of Physics, University of Natal, King George VAvenue, Durban, Natal, South Africa
}

(Received 5 September 1985; accepted for publication 4 April 1986)

\begin{abstract}
We define, in analogy to electrodynamics, the gravitational Poynting vector and show that it provides a mechanism for the transfer of gravitational energy to a system of falling objects.
\end{abstract}

By considering a special system (i.e., a point mass moving in the gravitational field of a line mass) it is easily demonstrated on relativistic grounds that the Newtonian gravitational interaction between masses appears in two distinct though interrelated forms. 'In addition to the well-known static interaction $\mathbf{F}=m \mathbf{g}$, where for instance $g=-G M /$ $r^{2}$ for a point mass $M$ ( $G$ is the gravitational constant) there is a "gravinetic" interaction between moving masses $\mathbf{F}=m \mathbf{v} \times \mathbf{b}$, where $\mathbf{v}$ is the velocity of the mass $m$, moving through the "gravinetic" field $b$. For a point mass $M$ moving with velocity $\mathbf{V}$ this field takes the form $\mathbf{b}=-(G /$ $\left.c^{2}\right)\left(M V \times \hat{\mathbf{r}} / r^{2}\right)$, where $\hat{\mathbf{r}}$ is a unit vector in the direction of $r$, which points from the moving mass $M$ to the field point.

The existence of a velocity-dependent gravitational interaction is well known from the general theory of relativity: the off-diagonal elements of the gravitational field tensor are indeed velocity dependent, but are usually dismissed as insignificant in the weak field approximation. ${ }^{2}$ Furthermore, the fact that gravinetic forces appear as a consequence of the special theory of relativity, although briefly mentioned in some textbooks on electrodynamics, ${ }^{3}$ has not penetrated into the basic literature on mechanics and gravity. This is an unfortunate omission, particularly since it could serve as an accessible introduction to some features of General Theory. In this paper we would like to point out that the "gravitational Poynting vector," formed in analogy to the electromagnetic one, is significant, and

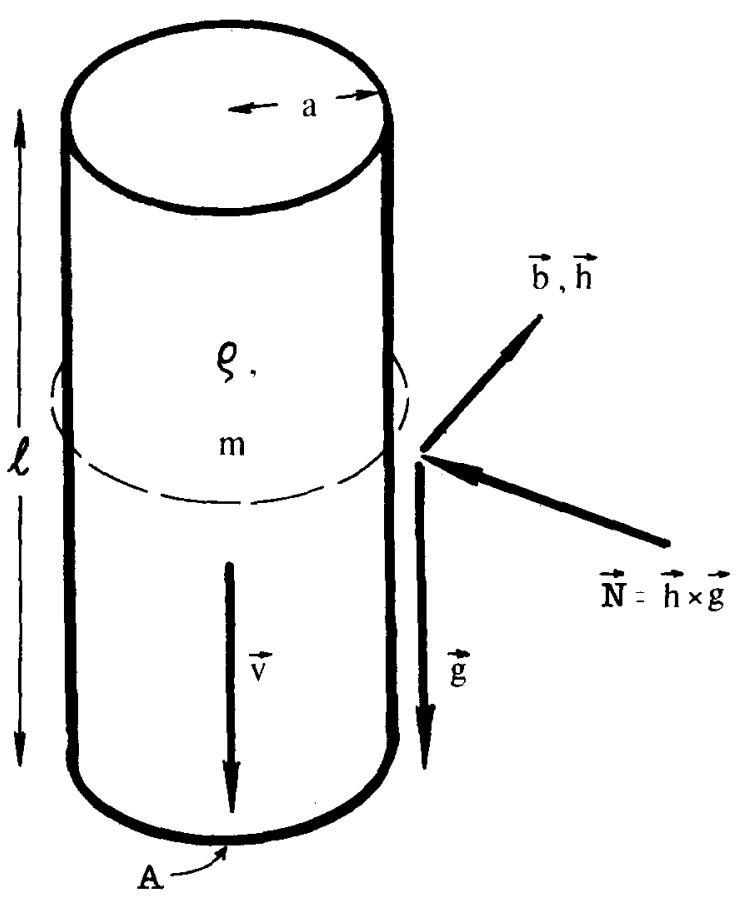

Fig. 1. The cylinder, mass $m$, falling with velocity $\mathbf{v}$ in the gravitational field $\mathbf{g}$. 